\title{
Does the Sun have a subsolar metallicity?
}

\author{
Martin Asplund \\ Max Planck Institute for Astrophysics, Postfach 1317, D-85741 Garching b. München, \\ Germany \\ email: asplund@mpa-garching.mpg.de
}

\begin{abstract}
The solar chemical composition has recently undergone a drastic revision, in particular in terms of the $\mathrm{C}, \mathrm{N}, \mathrm{O}$ and $\mathrm{Ne}$ abundances that have been lowered by almost a factor of two. In this invited review I will describe the different compounding reasons for this change (3D model atmospheres, non-LTE line formation, improved atomic/molecular data) and discuss some astrophysical implications thereof, which fall under both good (solar neighborhood) and bad (helioseismology) news. The most recent literature regarding the solar $\mathrm{O}$ abundance is surveyed and a critical evaluation whether or not these support the low solar abundance scale is presented. Finally I venture to make some predictions to what the real solar O abundance may be.
\end{abstract}

Keywords. Convection, line: formation, radiative transfer, Sun: abundances, Sun: atmosphere, Sun: granulation

\section{Introduction}

The solar chemical composition is a fundamental yardstick in astronomy, to which the elemental abundances of essentially all cosmic objects, be it planets, stars, nebulae or galaxies, are anchored. The importance of having accurate solar elemental abundances can thus not be overstated. From the pioneering efforts of Russell (1929) and Suess \& Urey (1956) to the more recent works of Anders \& Grevesse (1989), Grevesse \& Sauval (1998), Lodders (2003) and Asplund et al. (2005a), compilations of the solar system abundances have had, and will no doubt continue to have, an extremely wide-ranging use in astronomy and cosmology. To illustrate this obvious point, it here suffices to mention that Anders \& Grevesse (1989) is currently the fourth most cited astronomy article of all time according to the ADS database.

There are two independent and complementary ways of determining the solar system abundances, each with its pros and cons. Through mass spectroscopy of meteorites in terrestrial laboratories it is possible to directly measure the abundances of essentially all elements, including their various isotopes, with remarkable precision. Even in the most pristine meteorites - the so-called $\mathrm{C} 1$ chondrites that have been the least modified by various physical and chemical processes over the past $4.5 \mathrm{Gyr}$ - the volatile elements, including the most abundant elements hydrogen, helium, carbon, nitrogen, oxygen and neon, have been depleted to various degrees. As a consequence, it is not possible to rely on meteorites to determine the primordial solar system abundances for these elements. This also implies that one must measure all meteoritic abundances relative to another element than hydrogen, traditionally chosen to be silicon, and prior knowledge of the solar photospheric $\mathrm{Si}$ abundance is therefore required in order to place the meteoritic abundances on the same absolute scale as the Sun (Asplund 2000). With the exception of lithium and possibly beryllium depletion and a general $\sim 10 \%$ modification due to diffusion and gravitational settling, the solar photospheric abundances today reflect those present at 
the time of the birth of the solar system. On the other hand, the solar abundances can not obviously be determined with the same accuracy as for meteorites, in particular very little isotopic abundance information is available. Furthermore, the abundances are not observed but inferred: the solar spectrum must be interpreted using realistic models of the solar atmosphere and the spectrum formation process.

Recently the recommended solar atmospheric abundances of in particular $\mathrm{C}, \mathrm{N}, \mathrm{O}$ and $\mathrm{Ne}$ - the four most abundant elements next to $\mathrm{H}$ and $\mathrm{He}$ - have undergone a dramatic downward revision (Asplund et al. 2004, 2005a,b) While very welcome news in many, if not most, areas of astronomy, this lowering of the solar metal content from the canonical $2 \%$ to just over $1 \%$ (thus provoking the oxymoron in the title of this article) has caused a great deal of consternation for model builders of the solar interior and helioseismologists (e.g. Basu \& Antia 2008, and references therein). In this review I will briefly describe the underlying reasons for the revised abundances and present arguments in favour of the new analyses as well as discuss some lingering doubts about their reliability. I will also focus on a number of papers related to the solar oxygen abundance that have appeared very recently before making some cautionary predictions what the real solar abundances may be.

\section{Ingredients for solar spectroscopic analyses}

The most basic ingredient for any solar abundance analysis is an observed solar spectrum, either intensity at the center of the solar disk (Neckel \& Labs 1984) or flux (Kurucz et al. 1984). To minimize the effects of magnetic activity, the disk-center intensity spectrum used is obtained for the quiet Sun, i.e. for the typical solar granulation; for disk-integrated flux spectra the influence of solar activity, sunspots etc is still minimal in terms of derived abundances. Somewhat surprisingly, the available solar atlases sometimes differ and therefore yield slightly different abundances for some lines (e.g. Caffau et al. 2008). Whether the reasons for this can be traced to actual temporal variations in the solar atmosphere over the area averaged for the solar atlas or to subtle differences in for example the terrestrial absorption and data reduction is not yet clear. A renewed effort in acquiring new high-quality solar disk-center and flux atlases addressing these issues should be undertaken (Kurucz 2006).

To model the solar spectrum one then needs a solar model atmosphere. Traditionally various $1 \mathrm{D}$ hydrostatic models have been employed for the purpose. These come in two flavours, theoretical models such as the Kurucz (1993), PHOEnIX (Hauschildt et al. 1999) and MARCs (Gustafsson et al. 2008) grids, and semi-empirical models where perhaps the best known ones are the Holweger \& Mueller (1974) and VAL3C (Vernazza et al. 1976 ) versions. The Holweger \& Müller model has generally been the favoured model atmosphere of solar abundance aficionados, since its inferred temperature stratification from observed Fe I lines and continuum center-to-limb variation has been believed to be an accurate representation of the photospheric structure. All $1 \mathrm{D}$ modelling requires the use of the ad-hoc free parameters micro- and macroturbulence to obtain reasonable spectral line profiles; for theoretical models one also need to specify the mixing length parameters.

More recently 3D hydrodynamical models of the solar surface convection and atmosphere have become available (e.g. Stein \& Nordlund 1998; Asplund et al. 2000a; Freytag et al. 2002; Nordlund et al. 2008) and applied for solar abundance purposes (e.g. Asplund et al. 2000b, 2004, 2005b; Caffau \& Ludwig 2007; Caffau et al. 2008; Ayres 2008). In this modelling the standard hydrodynamical equations of conservation of mass, momentum and energy are solved together with the $3 \mathrm{D}$ radiative transfer equation in a 
small representative volume of the solar atmosphere typically covering $\sim 10$ granules at any given time. The 3D spectral line formation can then be computed with these models and the resulting spatially and temporally averaged line profiles compared with observations (see Asplund 2005, and references therein). While allowing for a self-consistent hydrodynamical description of convection makes for more sophisticated modelling, it does not necessarily imply more realistic outcomes, since approximations have to be made for computational reasons, in particular regarding the radiative transfer in which until now opacity binning has been used instead of opacity sampling. Therefore predictions from the 3D models have been confronted with a large number of observational diagnostics, in particular for the Sun. The 3D model successfully reproduce the solar granulation pattern in terms of length- and time-scales, brightness contrast, velocities etc (Nordlund et al. 2008). Perhaps the most impressive agreement comes from a comparison of the detailed line shapes: without invoking any micro- and macroturbulence, the theoretical 3D line profiles are in essentially perfect agreement with the observed lines for a wide range of elements and transitions (Asplund et al. 2000a). In fact even the observed line shifts and asymmetries are extremely well reproduced in general. The line profiles are the intricate result of the atmospheric inhomogeneities and (anti-)correlations between temperature, density and velocity and are therefore very sensitive probes of the atmospheric conditions.

A problem with the 3D model of Asplund et al. (2000a) it that it appears to predict a slightly too steep temperature gradient as inferred from continuum center-to-limb variation (Ayres et al. 2006; Koesterke et al. 2008). As seen in Fig. 2 performs better than any available 1D theoretical model but not quite as well as for the Holweger \& Mueller (1974) model, which is not surprising since the latter was after all constructed in order to fulfill this observational constraint. The 3D CO5BOLD model employed by Caffau et al. (2008) performs as well as the Holweger \& Mueller (1974) model in this regard but it has not yet been extensively tested in respect to for example line profiles and asymmetries. The center-to-limb variation is however not the only probe of the atmospheric temperature stratification. The wings of the hydrogen Balmer lines are formed in roughly similar depths but these seemingly paint a conflicting picture. The 3D model of Asplund et al. (2000a) yield slightly too strong wings when assuming LTE, which may be rectified when accounting for departures from LTE (Barklem 2007b). The Holweger \& Mueller (1974) model, however, yields much too weak wings of $\mathrm{H} \beta$ even in LTE, which implies that its temperature gradient is too shallow in these layers (Pereira et al., in preparation). Which model atmosphere has the most realistic temperature structure is thus not yet settled.

In addition to a model of the solar atmosphere, one also needs a model for how the spectrum formation proceeds, in particular the line formation. For late-type stars such as the Sun, this basically boils down whether or not local thermodynamic equilibrium (LTE) is a valid assumption. In general, the collisional rates do not dominate sufficiently over the corresponding radiative rates in the solar atmosphere to ensure that LTE holds. While most elements and transitions do show departures from LTE in the Sun, in terms of abundance corrections the non-LTE effects are typically rather modest $(\leqslant 0.1 \mathrm{dex})$ (see Asplund 2005, and references therein). Some well-used solar lines, however, are significantly more affected, such as most O I lines.

Finally, all solar analyses rely heavily on having reliable input physics data. For the construction of model atmospheres, essential ingredients are equation-of-state and continuous and line opacities. For the line formation the most obvious data needed are oscillator strengths for the lines in question but a large number of other transition properties are also required which can affect the derived abundances, including the line broadening, partition functions, molecular dissociation energies etc. Moving to non-LTE vastly increases 


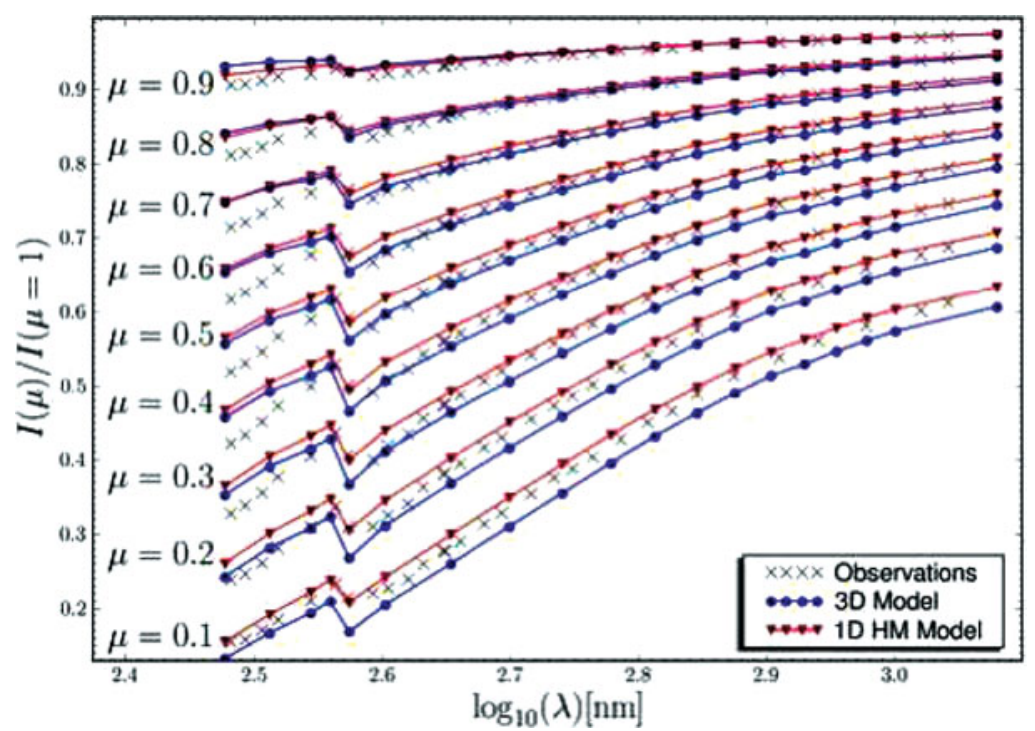

Figure 1. A comparison of the observed solar continuum center-to-limb variation (Neckel \& Labs 1984) (crosses) with the predictions for the Holweger \& Mueller (1974) 1D semi-empirical model (triangles) and the 3D hydrodynamical model of Asplund et al. (2000a) (circles). It can be seen that the observations fall in between the two theoretical curves, although with a slight preference for the Holweger \& Mueller (1974) model for $\mu \leqslant 0.4$. The shortcomings at the shortest wavelengths are probably related to the observations including the effects of lines.

the data requirements, since these need to be specified for all radiative transitions in the element under consideration as well as photoionization and collisional cross-sections. The situation is continuously improving through the heroic efforts of a relatively small number of atomic physicists, especially in regards to transition probabilities (e.g. Kurucz 1993; Plez 1998; Johansson et al. 2003; Lawler et al. 2006) and photoionization rates (e.g. Badnell et al. 2005) The progress for the important collisional data is less satisfactory in spite of some recent advances (Barklem et al. 2003; Barklem 2007a). Non-LTE calculations therefore still normally employ various recipes based on classical physics for electron and hydrogen collisions, often with a scaling factor somehow calibrated. For late-type stars arguably the biggest piece missing is reliable estimates for the inelastic hydrogen collisions (see discussion in Asplund 2005).

\section{The new solar chemical composition and implications thereof}

The 3D hydrodynamical solar model atmosphere has been the foundation for a reanalysis of the solar chemical composition that has been detailed in a series of papers (e.g. Asplund et al. 2000b, 2004, 2005a,b; Asplund 2000, 2004; Allende Prieto et al. 2001, 2002; Scott et al. 2006). The most noteworthy result of this work is no doubt the significant downward revision of the present-day solar photospheric C, N, O and Ne abundances by almost a factor of two compared with the widely used recommendations by Anders \& Grevesse (1989): $\log \epsilon_{\mathrm{C}}=8.39 \pm 0.05, \log \epsilon_{\mathrm{N}}=7.78 \pm 0.06, \log \epsilon_{\mathrm{O}}=8.66 \pm 0.05$, and $\log \epsilon_{\mathrm{Ne}}=7.84 \pm 0.06$. Besides employing a $3 \mathrm{D}$ rather than a $1 \mathrm{D}$ model, departures from LTE have been considered for transitions suspected to be affected, including performing full 3D non-LTE calculations for elements like O I. Furthermore, the best possible atomic and molecular data have been utilized and a careful evaluation of the solar spectrum 
was carried out to only include the most reliable lines the least affected by blends. It is important to remember that the driving force behind the new low solar abundances is not only the application of a 3D solar model but also allowance for non-LTE effects, identification of significant blends and improved line transition data. Indeed for some lines the 3D effects are very small yet a consistent low abundance is obtained from them for other reasons.

Allende Prieto et al. (2001) and Asplund et al. (2004) employed a wide range of abundance indicators to determine the solar $\mathrm{O}$ abundance: low excitation forbidden [O I] lines, high-excited permitted $\mathrm{O}$ I lines and $\mathrm{OH}$ lines in the infrared from both the vibrationrotation and pure rotation bands; Scott et al. (2006) added CO lines to conclude that the ${ }^{16} \mathrm{O} /{ }^{18} \mathrm{O}$ isotopic ratio is identical to within the uncertainties to the terrestrial value. Since the various transitions have vastly different sensitivities to the atmospheric conditions and the line formation processes, achieving consistent abundances from them is a very strong test of the accuracy of the analysis. Traditionally $1 \mathrm{D}$-based analyses have implied a significantly higher $\mathrm{O}$ abundance from the $\mathrm{OH}$ and $[\mathrm{O} \mathrm{I}]$ lines than for $\mathrm{O}$ I when non-LTE effects are considered for the latter (Asplund et al. 2004). The presence of temperature inhomogeneities and a cooler mean temperature stratification in the 3D model compared with for example the Holweger \& Mueller (1974) model result in significant reduction in the $\mathrm{OH}$-based results. Furthermore, Allende Prieto et al. (2001) demonstrated that the crucial [O I] $630 \mathrm{~nm}$ line is blended by a Ni I line, which was subsequently confirmed experimentally (Johansson et al. 2003). The end result is that finally all $\mathrm{O}$ indicators agree, which is a strong argument in favour of the new value. The solar $\mathrm{Ne}$ and $\mathrm{Ar}$ abundances are similarly affected, since they are based on the coronal O/Ne and $\mathrm{O} / \mathrm{Ar}$ ratios together with the photospheric $\mathrm{O}$ abundance.

In the case of $\mathrm{C}$, even more diagnostics are available: $[\mathrm{CI}], \mathrm{C}$, $\mathrm{CH}$ vibration-rotation, $\mathrm{CH}$ electronic, $\mathrm{C}_{2}$ and $\mathrm{CO}$ lines. Again, with any $1 \mathrm{D}$ model these imply $\mathrm{C}$ abundances differing by $0.2-0.3$ dex, while the $3 \mathrm{D}$-based result are the same to within 0.1 dex (Allende Prieto et al. 2002; Asplund et al. 2005a,b; Scott et al. 2006). Given the very different formation depths and temperature and pressure sensitivities for the various transitions, this excellent agreement is very gratifying. Compared with the recommendation of Anders \& Grevesse (1989) the new value is 0.17 dex lower. The reasons for the downward revision are manifold also for C: 3D effects in particular for the molecules, non-LTE effects for $\mathrm{C}$, blends and better atomic/molecular data. Using CO lines in the infrared, Scott et al. (2006) found that the solar photospheric ${ }^{12} \mathrm{C} /{ }^{13} \mathrm{C}$ ratio is in agreement with the telluric value.

Given that C, N, O and Ne are the most abundant elements in the Universe besides $\mathrm{H}$ and $\mathrm{He}$, it is not surprising that the new solar abundances of Asplund et al. (2005a) have had a wide-ranging impact in astronomy. Naturally this affects the abundances of other cosmic objects when using the customary square-bracket notation in astronomy, such as $[\mathrm{C} / \mathrm{H}]$ and $[\mathrm{O} / \mathrm{Fe}]$. For the Sun, the mass fraction of metals $Z$ decreases to from 0.0194 to 0.0122 . One of the good news is that the Sun is no longer peculiar in terms of its apparent metal-richness compared to its surroundings: the new solar abundances are in excellent agreement with what is measured for nearby OB stars (e.g. Przybilla et al. 2008) and the local interstellar medium (e.g. Esteban et al. 2005), in particular when factoring in the overall $\approx 0.04$ dex reduction in the photospheric metal abundances due to diffusion (Turcotte et al. 1998) and the expected $\approx 0.05$ dex chemical enrichment of the interstellar medium over the past 4.5 Gyr (Chiappini et al. 2003).

The bad news is that the revised solar chemical composition messes up the predicted solar interior structure, since C, N, O and Ne are significant contributors to the opacity. The predicted sound speed as a function of depth is therefore altered and no longer agree 
as nicely with observations as inferred from helioseismology (e.g. Basu \& Antia 2008, and references therein). This discrepancy has been named the solar modelling problem (or even the oxygen crisis, which is clearly not an appropriate labelling) and has received a great deal of attention lately. A large number of solutions have been put forward but most have already been ruled out, leaving only very few as viable explanations. Initially it was believed by some that missing opacity would do the trick but the very significant amount required (10-20\%) have not been forthcoming in more recent calculations (Badnell et al. 2005). Another possibility is that a $\sim 0.5$ dex increase in the solar Ne abundance compensates for in particular the lower $\mathrm{O}$ abundance, a suggestion that has received some empirical support (Drake \& Testa 2005). More recent works, however, suggest that although the solar neighborhood Ne abundance may be higher than the solar value of Asplund et al. (2005a), it is not enough by itself to solve the problem (Cunha et al. 2006; Morel \& Butler 2008; Przybilla et al. 2008; Robrade et al. 2008). Perhaps the only proposed explanation still standing is the effects of internal gravity waves, which may act as an effective opacity source and provide additional mixing (Arnett et al. 2005; Charbonnel \& Talon 2005). No quantitative estimate of this effect has of yet appeared in the literature.

The stubbornness with which this solar modelling problem has refused to yield a solution has lead some to question the new solar abundances of Asplund et al. (2005a), rather than the physical ingredients of the solar interior models, in spite of the successes of the new 3D-based spectroscopic analyses briefly outlined above. A significantly better agreement with helioseismology is obtained when instead using the (preliminary) abundances recommended by Grevesse \& Sauval (1998) obtained from an empirical and somewhat ad-hoc additional adjustment of the temperature structure of the Holweger \& Mueller (1974); in this context it is perhaps worth noting that even then the discrepancy is much larger than expected purely from the helioseismic statistical and systematic errors and that the largest problem occur at the same location, namely immediately below the solar convection zone. A further revisit of the solar abundance issue is, however, clearly justified given the current state of affairs.

\section{Recent developments}

In this section I will review some very recent works on the solar oxygen abundance and discuss how they agree or disagree with the low solar $\mathrm{O}$ abundance advocated by Asplund and collaborators; unfortunately the solar $\mathrm{C}$ and $\mathrm{N}$ abundances have been largely overlooked in this respect.

\subsection{Ayres (2008)}

Ayres (2008) has carried out an analysis of the [O I] $630 \mathrm{~nm}$ line using one snapshot of a 3D CO5BOLD solar atmosphere model. He followed the same procedure as Allende Prieto et al. (2001) and Asplund et al. (2004) for this line, namely to allow the Ni blend to vary freely in obtaining the best overall line profile fit. In many ways this new study agrees well with that of Allende Prieto et al. but differs in one critical respect: the derived O abundance, which here is found to be $\log \epsilon_{\mathrm{O}}=8.81 \pm 0.04$, or 0.12 dex higher. Part of this difference must stem from the different temperature gradients in the line-forming region of the two 3D models (Caffau et al. 2008) but it is not yet clear if this is the whole explanation. A serious problem is presented by the implied solar Ni abundance, since Ayres find that the best fit is obtained with a $\mathrm{Ni}$ line strength of only $70 \%$ of the theoretical line strength based on the accurate experimental $g f$-value of Johansson et al. (2003) and the Ni abundance of Grevesse \& Sauval (1998). This difference exceeds 
the quoted uncertainties in these two values. In terms of abundance, a 0.15 dex lower $\mathrm{Ni}$ abundance corresponds to an increase of the derived $\mathrm{O}$ abundance by $\approx 0.09$ dex for disk-center intensity (Allende Prieto et al. 2001; Caffau et al. 2008), which would reduce the suggested value of Ayres (2008) to $\approx 8.72$ but at the expense of a deteriorated profile fit. It remains to be determined if this is an acceptable trade-off.

\subsection{Caffau et al. (2008)}

Caffau et al. (2008) have studied both [O I] and O I lines using the same 3D CO5BOLD model as employed by Ayres (2008) but covering in total 19 snapshots. Their recommended solar $\mathrm{O}$ abundance from the weighted mean of all lines from both flux and diskcenter intensity spectra is $\log \epsilon_{\mathrm{O}}=8.76 \pm 0.07$, i.e. roughly half-way between the values of Grevesse \& Sauval (1998) (log $\left.\epsilon_{\mathrm{O}}=8.83\right)$ and Asplund et al. (2005a) ( $\left.\log \epsilon_{\mathrm{O}}=8.66\right)$.

For the [O I] $630 \mathrm{~nm}$ line, Caffau et al. includes the Ni blend at full strength in contrast to Ayres (2008) and consequently find a much smaller $\mathrm{O}$ abundance: $\log \epsilon_{\mathrm{O}}=8.68$. The value itself is in excellent agreement with Allende Prieto et al. (2001) but is partly due to their larger adopted contribution of the Ni line to the $630 \mathrm{~nm}$ feature. They also found that the even weaker [O I] line at $636 \mathrm{~nm}$, which is blended by CN lines and a Ca autoionization line, imply a 0.1 dex higher abundance; Asplund et al. (2004) found the two lines to give identical abundances. The reason for this discrepancy in the Caffau et al. study has not yet been fully identified.

For the O I lines, they carry out 3D LTE calculations to which non-LTE abundance corrections computed with 1D models are applied, in contrast to Asplund et al. (2004) who performed a full 3D non-LTE study; the 1D and 3D non-LTE effects are, however, quite similar so this simplification should not bias the results significantly. The O I-based result of Caffau et al. is 0.12 dex higher than the corresponding value in Asplund et al.. Most of this difference can be traced to different assumptions regarding the poorly known inelastic $\mathrm{H}$ collisions for the non-LTE calculations and the adopted equivalent widths with a smaller contribution coming from selection and weighting of lines. In fact when the same input data are used the two studies agree remarkably well for most of the O I lines, in spite of two completely independent 3D models and line formation codes have been used. As a corollary it follows that the outcome for these lines is quite insensitive to the choice of model atmosphere, be it 3D or 1D, but is driven more by other factors, in particular departures from LTE. This is also demonstrated by Socas-Navarro \& Norton (2007) who derived a 3D semi-empirical solar model from inversion of spatially resolved observations of two Fe I line profiles akin to the Holweger \& Mueller (1974) model constructed from spatially averaged spectra; their O I $777 \mathrm{~nm}$-based abundance is in excellent agreement with the value of Asplund et al. (2004), since neither considered H collisions in their non-LTE studies based on the available laboratory and quantum mechanical evidence for other species (see discussion in Asplund 2005). Caffau et al. on the other hand make use of the classical Drawin (1968) formula with a scaling factor $S_{H}=1 / 3$, apparently largely adopted as a middle-ground between the "extremes" $S_{H}=0$ and 1 .

Allende Prieto et al. (2004) argued on the basis of the observed center-to-limb variation in the solar O I $777 \mathrm{~nm}$ line profiles that $S_{H}=1$ is slightly preferable over having no $\mathrm{H}$ collisions $\left(S_{H}=0\right)$ while the LTE case could be ruled out at very high significance; the $S_{H}=1 / 3$ case was not considered. It is important to realize that such empirical calibrations of $S_{H}$ is certainly no substitute to having real quantum mechanical or laboratory estimates, since the necessary thermalization may well come from other atomic processes than $\mathrm{H}$ collisions, as in the case of charge transfer reactions in $\mathrm{Li}$ (Barklem et al. 2003). Furthermore, the observations of Allende Prieto et al. were not optimal. Pereira et al. (in preparation) have tried to remedy this by observing closer to the limb 


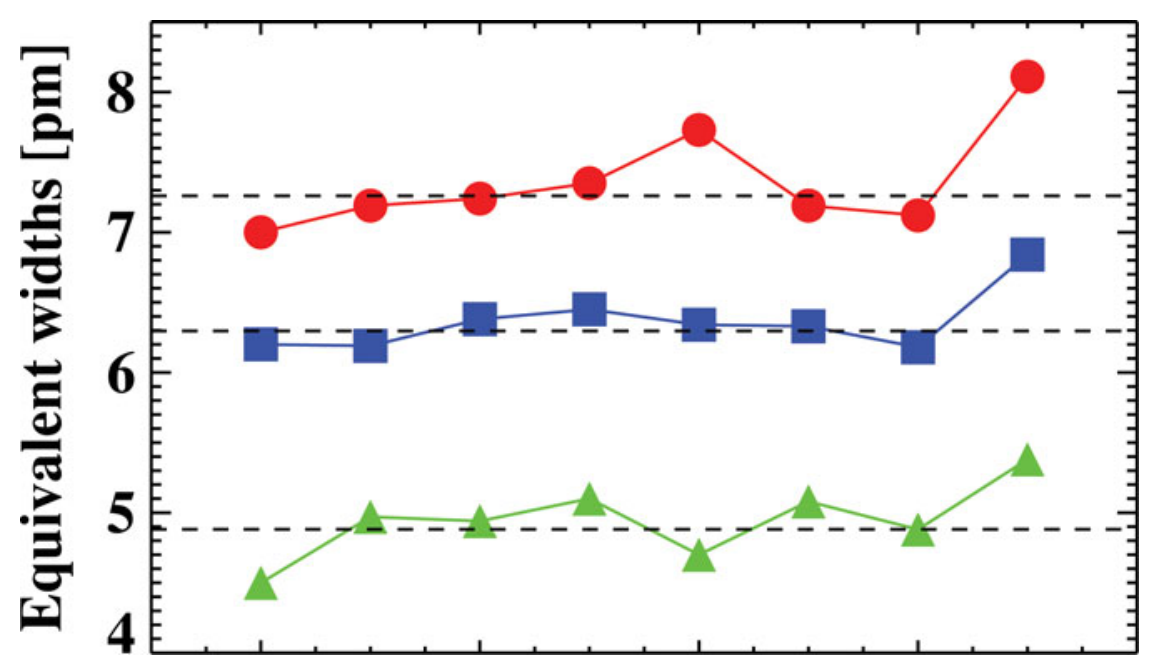

Figure 2. The published flux equivalent widths of the O I $777 \mathrm{~nm}$ triplet given in the eight influential $\mathrm{O}$ abundance analyses over the past three decades. From left to right the symbols correspond to in chronological order: Lambert (1978), Biemont et al. (1991), Kiselman (1993), Reetz (1999), Holweger (2001), Asplund et al. (2004), Allende Prieto et al. (2004) and Caffau et al. (2008); for those studies only presenting disk-center intensity equivalent widths they have been converted to corresponding flux values using the ratio of intensity to flux line strengths. The Caffau et al. equivalent widths are significantly higher than the other published values for unknown reasons. The dashed lines denote the mean for the three lines when excluding the Caffau et al. values.

and using a more suitable spectrograph. In addition they have obtained spatially resolved spectra of both the $777 \mathrm{~nm}$ and other $\mathrm{O}$ lines to test the 3D models and line formation; the analysis is currently ongoing. H. Ludwig et al. (private communication) have carried out a similar study using their own observations and find a preference for $S_{H}=0$. In terms of abundance, having no $\mathrm{H}$ collisions would lower the Caffau et al. $\mathrm{O}$ abundance by $\approx 0.03$ dex.

Recently, new cross-sections have been computed for electron collisions with O I (Barklem 2007a). Fabbian et al. (2008) find that the non-LTE effects become slightly more severe for the Sun when including the new collisional data, which corresponds to a lowering of the derived abundance by $\approx 0.02$ dex.

Surprisingly the largest difference between the Caffau et al. and Asplund et al. studies can be traced to the adopted line strengths. In contrast to Asplund et al., Caffau et al. did not carry out full 3D non-LTE calculations and therefore had to rely on equivalent widths to derive the $\mathrm{O}$ abundances (the quoted equivalent widths given in Asplund et al. are the theoretical values from the best fitting 3D non-LTE profiles). Measuring equivalent widths is often tricky and subjective, in particular for the strong, partly saturated $777 \mathrm{~nm}$ triplet with continuum placement, exact line shape and which wavelength region to integrate over some of the key factors to worry about. Furthermore, Caffau et al. found a disconcerting difference between the available solar atlases already alluded to above (which of course would also equally affect any attempts to derive abundances from profile fitting). The equivalent widths of the $777 \mathrm{~nm}$ lines adopted by Caffau et al. are much larger than any other values in the literature. As demonstrated in Fig. 4.2, the Caffau et al. values differ by $\geqslant 3 \sigma$ compared with the seven most recent studies. By itself this does not obviously invalidate their adopted equivalent widths but it is paramount 
to try to understand why the values are systematically higher than previously found. Independent new remeasurements have yielded equivalent widths in between the Caffau et al. values and the mean shown in Fig. 4.2 (N. Grevesse and J. Meléndez, private communication). More work is needed to resolve this issue. In the meantime, it is clear that profile fitting offers a superior method in determining abundances since the detailed line shape, including the asymmetries, are automatically accounted for. In terms of abundance, adopting instead the Asplund et al. predicted line strengths would lower the Caffau et al. abundances for the $777 \mathrm{~nm}$ triplet by $\approx 0.09 \mathrm{dex}$.

In summary, Caffau et al. (2008) found a slightly higher O abundance than Asplund et al. (2004) but the differences basically vanish when the same input data are adopted. It remains to be convincingly demonstrated that their choices in this respect are preferable with some answers expected in the near future.

\subsection{Centeno \& Socas-Navarro (2008)}

Centeno \& Socas-Navarro (2008) have invented a novel method, which to my knowledge has not previously been employed in deriving abundances. Instead of using a normal solar spectrum they analyse spectropolarimetric sunspot observations of the blend of [O I] and $\mathrm{Ni}$ I at $630 \mathrm{~nm}$. They estimated an atomic ratio of $\mathrm{O} / \mathrm{Ni}=210 \pm 24$ from the line asymmetry of the Stokes $V$ profile using their own semi-empirical model atmosphere of the sunspot obtained from an inversion of nearby Fe I lines; adopting the more traditional sunspot model of Maltby et al. (1986) would lower the ratio to $\approx 175$. The total $\mathrm{O}$ abundance was found to be $\log \epsilon_{\mathrm{O}}=8.86 \pm 0.07$ after applying the Ni abundance of Grevesse \& Sauval (1998) and correcting for the $\sim 50 \%$ of O locked up in CO in the cool environments of sunspots, i.e. even slightly higher than the recommended value of Grevesse \& Sauval (1998).

A few factors that could influence the final derived abundance should be borne in mind though. Firstly, Centeno \& Socas-Navarro (2008) adopted an outdated value for the $[\mathrm{O} \mathrm{I}] g f$-value that is 0.06 dex too low. Secondly, converting from atomic $\mathrm{O}$ to total $\mathrm{O}$ content requires prior knowledge of the $\mathrm{C}$ abundance. If it is true as they argue that all other molecules other than CO can be ignored, the maximum correction is obtained when assuming that all $\mathrm{C}$ is in the form of $\mathrm{CO}$, which means that adopting instead the $\mathrm{C}$ abundance of (Asplund et al. 2005b) would imply a lowering of the derived O abundance. Thirdly, Centeno \& Socas-Navarro made use of the solar Ni abundance of Grevesse \& Sauval (1998), while a more recent, 3D-based analysis suggest a value $\approx 0.08$ dex lower (Scott et al., in preparation). Taken together, the Stokes $V$ profile of the [O I] $630 \mathrm{~nm}$ line could thus equally well support an $\mathrm{O}$ abundance of $\log \epsilon_{\mathrm{O}}=8.71$ (or 8.69 had the Maltby et al. sunspot model been used).

While the current observations and analysis are quite inconclusive, this new and interesting alternative approach clearly has a great deal of potential and should be studied more. In this context, it would be very valuable if a similar spectropolarimetric analysis could be performed for features coming from other elements, whose solar photospheric abundances are less in dispute than $\mathrm{O}$ in order to confirm that the method yields consistent results with traditional solar spectroscopy.

\subsection{Meléndez \& Asplund (2008)}

Meléndez \& Asplund (2008) have employed a forbidden O line previously not used in solar spectroscopy, namely the [O I] $557.7 \mathrm{~nm}$ transition. Because the line is heavily blended by two $\mathrm{C}_{2}$ lines (Fig. 4.4), it has before not been considered a reliable abundance indicator. 


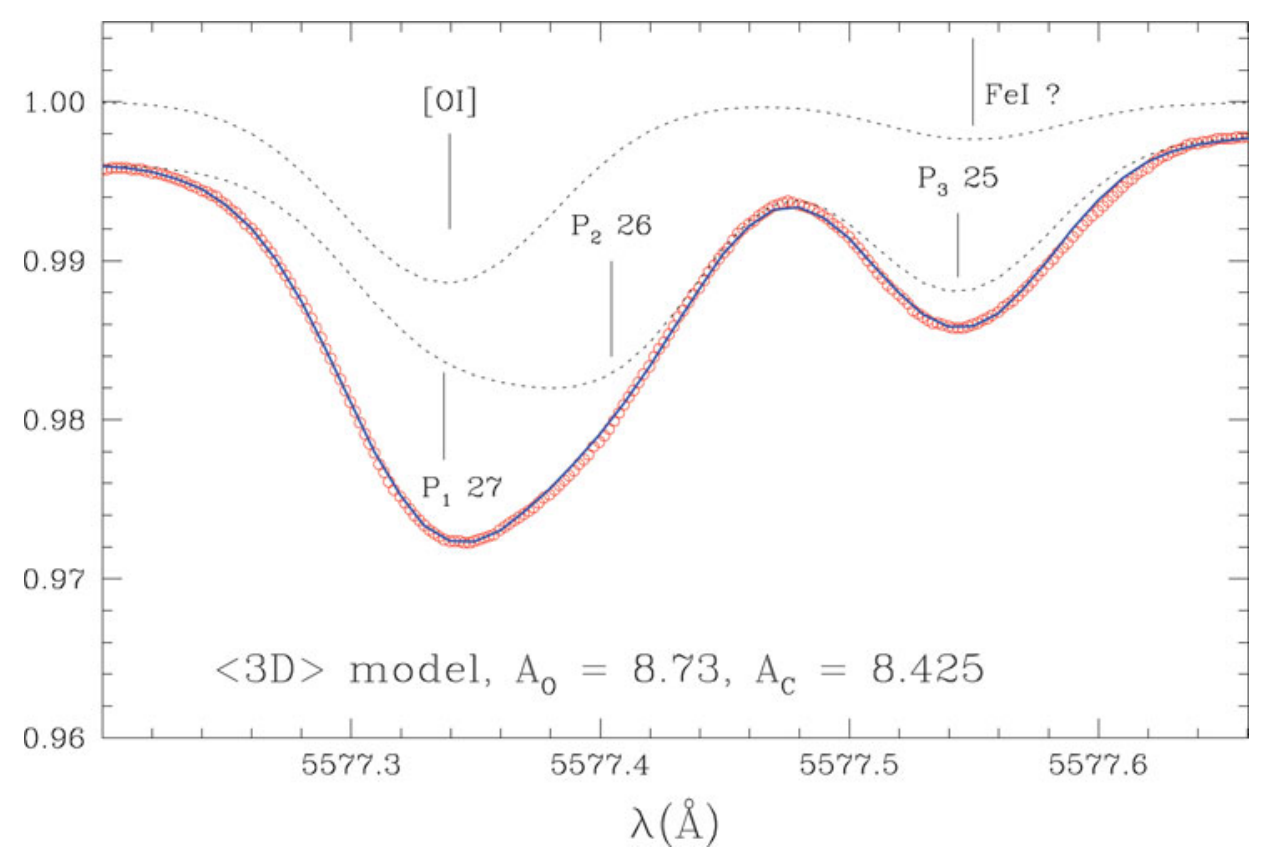

Figure 3. The solar disk-center forbidden [O I] $557.7 \mathrm{~nm}$ line (circles) together with the theoretical profile using the horizontally averaged 3D model assuming $\log \epsilon_{\mathrm{O}}=8.73$ and $\log \epsilon_{\mathrm{C}}=8.42$. Also shown with dotted lines are the contributions from the $[\mathrm{O} \mathrm{I}]$ and $\mathrm{C}_{2}$ lines individually. Figure taken from Meléndez \& Asplund (2008).

Meléndez \& Asplund have shown, however, that the strength of the $\mathrm{C}_{2}$ lines can be accurately constrained by numerous other nearby $\mathrm{C}_{2}$ lines from the same molecular band with essentially identical excitation potential, line strengths and line formation properties; the expected uncertainties in relative line strengths between these $\mathrm{C}_{2}$ lines are very small indeed. Another advantage with this line is that it originates not from the $\mathrm{O}_{\mathrm{I}}$ ground state as the $\left[\mathrm{O}^{\circ} \mathrm{I}\right]$ lines at 630 and $636 \mathrm{~nm}$ but from a level at $2 \mathrm{eV}$, which makes it less sensitive to the atmospheric structure and thus the details of the model atmosphere Asplund (2005).

Relying on the same 3D hydrodynamical solar model as in Asplund et al. (2004), Meléndez \& Asplund derived $\log \epsilon_{\mathrm{O}}=8.70 \pm 0.08$ with very similar results coming from 1D models (e.g. 8.73 with the Holweger \& Mueller (1974) model) The mean abundance of the tested $3 \mathrm{D}$ and $1 \mathrm{D}$ models was found to be $\log \epsilon_{\mathrm{O}}=8.71 \pm 0.02$, i.e. in very good agreement with the Asplund et al. (2004) results for the other two [O I] lines.

\section{Does the Sun really have a subsolar metallicity?}

From the previous section, it is clear that there is currently no clear consensus what the solar $\mathrm{O}$ abundance, and by extension what the overall solar metal content, is. It is probably fair to say that nowadays there is no support for the high abundance $\log \epsilon_{\mathrm{O}}=$ 8.93 recommended by Anders \& Grevesse (1989) but the question is how much lower the real value is: is it as low as $\log \epsilon_{\mathrm{O}}=8.66$ as advocated by Asplund et al. (2004) or is it closer to $\log \epsilon_{\mathrm{O}}=8.83$ as the uncompleted study of of Grevesse \& Sauval (1998) suggested? It would be premature to here give a definite answer, since a great deal of work still remains to be done before the final tally is in. Nevertheless I will venture to 
here make some, not entirely well-grounded, speculations and extrapolations based on the available evidence presented above.

The three forbidden [O I] lines seem to suggest $\log \epsilon_{\mathrm{O}} \approx 8.7-8.75$, i.e. slightly larger than Asplund et al. (2004) due to a 3D model that even better reproduces the continuum center-to-limb variation but partly offset by an increased $\mathrm{Ni}$ contribution to the $630 \mathrm{~nm}$ line. The $\mathrm{O}$ I lines may be $\sim 0.05$ dex higher than in Asplund et al. following adjustments to the non-LTE abundance corrections and/or adopted line strength, which would result in $\log \epsilon_{\mathrm{O}} \approx 8.7$; the exact $3 \mathrm{D}$ atmospheric structure is not particularly important in dictating the outcome. Similarly one would expect the molecular-based abundances to increase with a slightly warmer temperature structure, which is consistent with the fact that in Asplund et al. the $\mathrm{OH}$ lines implied slightly lower $\mathrm{O}$ abundances than the atomic lines. An upper limit to the abundance is set by the Holweger \& Mueller (1974) model, since it has a slightly too shallow temperature gradient (Fig. 2) and the presence of temperature inhomogeneities inevitably lead to a lowering of the derived $\mathrm{O}$ abundance. Given that Asplund et al. found that the overall 3D effects for the $\mathrm{OH}$ lines are in roughly equal parts a combination of mean stratification and atmospheric inhomogeneities, the $\mathrm{OH}$-based abundance may be something like $\log \epsilon_{\mathrm{O}} \approx 8.7-8.75$.

In summary, it seems reasonable to expect the solar $\mathrm{O}$ abundance to be $\log \epsilon_{\mathrm{O}} \approx$ $8.7-8.75$, i.e. a slight upward revision of the value presented in Asplund et al. (2004, 2005a,b). Such an O abundance would ease but not remove the discrepancy between solar interior models and helioseismology. It should be borne in mind though that this is nothing more than my own personal expectation, which have not yet been confirmed or otherwise by actual calculations. We are currently working on constructing a new 3D solar model based on an improved treatment of radiative transfer, including the first ever models employing opacity sampling rather than opacity binning to treat the line-blanketing. Whether or not this model will fulfill the constraints from center-tolimb variation as well as the other tests such as detailed line profiles remains to be seen. A complete re-analysis of not only the solar $\mathrm{C}, \mathrm{N}$ and $\mathrm{O}$ abundances but of most elements accessible by solar spectroscopy is currently underway, with the results presented elsewhere (Asplund et al., in preparation).

\section{Acknowledgements}

It is a pleasure to acknowledge the wonderful work done by my main collaborators on the topic of solar modelling and abundance analysis: Carlos Allende Prieto, Mats Carlsson, Remo Collet, Nicolas Grevesse, Wolfgang Hayek, Dan Kiselman, David Lambert, Åke Nordlund, Jacques Sauval, Patrick Scott, Bob Stein and Regner Trampedach. Finally, the author is grateful to the editors for showing a great deal of patience when waiting for this contribution.

\section{References}

Allende Prieto, C., Asplund, M., \& Fabiani Bendicho, P. 2004, A\&A, 423, 1109

Allende Prieto, C., Lambert, D. L., \& Asplund, M. 2001, ApJL, 556, L63

Allende Prieto, C., Lambert, D. L., \& Asplund, M. 2002, ApJL, 573, L137

Anders, E. \& Grevesse, N. 1989, Geochim. Cosmochim., 53, 197

Arnett, D., Meakin, C., \& Young, P. A. 2005, in ASP Conf. Series, Vol. 336, 235

Asplund, M. 2000, A\&A, 359, 755

Asplund, M. 2004, A\&A, 417, 769

Asplund, M. 2005, ARA\&A, 43, 481 
Asplund, M., Grevesse, N., \& Sauval, A. J. 2005a, in ASP Conf. Series, Vol. 336, 25

Asplund, M., Grevesse, N., Sauval, A. J., Allende Prieto, C., \& Blomme, R. 2005b, A\&A, 431, 693

Asplund, M., Grevesse, N., Sauval, A. J., Allende Prieto, C., \& Kiselman, D. 2004, A\&A, 417, 751

Asplund, M., Nordlund, Å., Trampedach, R., Allende Prieto, C., \& Stein, R. F. 2000a, A\&A, 359,729

Asplund, M., Nordlund, Å., Trampedach, R., \& Stein, R. F. 2000b, A\&A, 359, 743

Ayres, T. R. 2008, ApJ, in press

Ayres, T. R., Plymate, C., \& Keller, C. U. 2006, ApJS, 165, 618

Badnell, N. R., Bautista, M. A., Butler, K., et al. 2005, MNRAS, 360, 458

Barklem, P. S. 2007a, A\&A, 462, 781

Barklem, P. S. 2007b, A\&A, 466, 327

Barklem, P. S., Belyaev, A. K., \& Asplund, M. 2003, A\&A, 409, L1

Basu, S. \& Antia, H. M. 2008, Physics Reports, 457, 217

Biemont, E., Hibbert, A., Godefroid, M., Vaeck, N., \& Fawcett, B. C. 1991, ApJ, 375, 818

Caffau, E. \& Ludwig, H.-G. 2007, A\&A, 467, L11

Caffau, E., Ludwig, H.-G., Steffen, M., et al. 2008, ArXiv astro-ph/0805.4398

Centeno, R. \& Socas-Navarro, H. 2008, ApJL, 682, L61

Charbonnel, C. \& Talon, S. 2005, Science, 309, 2189

Chiappini, C., Romano, D., \& Matteucci, F. 2003, MNRAS, 339, 63

Cunha, K., Hubeny, I., \& Lanz, T. 2006, ApJL, 647, L143

Drake, J. J. \& Testa, P. 2005, Nature, 436, 525

Drawin, H.-W. 1968, Zeitschrift fur Physik, 211, 404

Esteban, C., García-Rojas, J., Peimbert, M., et al. 2005, ApJL, 618, L95

Fabbian, D., Asplund, M., Carlsson, M., \& Kiselman, D. 2008, A\&A, in press

Freytag, B., Steffen, M., \& Dorch, B. 2002, Astronomische Nachrichten, 323, 213

Grevesse, N. \& Sauval, A. J. 1998, Space Science Reviews, 85, 161

Gustafsson, B., Edvardsson, B., Eriksson, K., et al. 2008, A\&A, 486, 951

Hauschildt, P. H., Allard, F., Ferguson, J., Baron, E., \& Alexander, D. R. 1999, ApJ, 525, 871

Holweger, H. 2001, in American Institute of Physics Conference Series, Vol. 598, , 23

Holweger, H. \& Mueller, E. A. 1974, Solar Physics, 39, 19

Johansson, S., Litzén, U., Lundberg, H., \& Zhang, Z. 2003, ApJL, 584, L107

Kiselman, D. 1993, A\&A, 275, 269

Koesterke, L., Allende Prieto, C., \& Lambert, D. L. 2008, ApJ, 680, 764

Kurucz, R. L. 1993, in ASP Conf. Series, Vol. 44, IAU Colloq. 138, ed. M. M. Dworetsky, F. Castelli, \& R. Faraggiana, 87

Kurucz, R. L. 2006, ArXiv astro-ph/0605029

Kurucz, R. L., Furenlid, I., Brault, J., \& Testerman, L. 1984, Solar flux atlas from 296 to 1300 $\mathrm{nm}$ (National Solar Observatory)

Lambert, D. L. 1978, MNRAS, 182, 249

Lawler, J. E., Den Hartog, E. A., Sneden, C., \& Cowan, J. J. 2006, ApJS, 162, 227

Lodders, K. 2003, ApJ, 591, 1220

Maltby, P., Avrett, E. H., Carlsson, M., et al. 1986, ApJ, 306, 284

Meléndez, J. \& Asplund, M. 2008, A\&A, submitted

Morel, T. \& Butler, K. 2008, ArXiv astro-ph/0806.0491

Neckel, H. \& Labs, D. 1984, Solar Physics, 90, 205

Nordlund, A., Stein, R., \& Asplund, M. 2008, Living Reviews in Solar Physics, in press

Plez, B. 1998, A\&A, 337, 495

Przybilla, N., Nieva, M. F., Heber, U., \& Butler, K. 2008, A\&A, submitted

Reetz, J. 1999, Astroph. \& Space Science, 265, 171

Robrade, J., Schmitt, J. H. M. M., \& Favata, F. 2008, A\&A, 486, 995

Russell, H. N. 1929, ApJ, 70, 11

Scott, P. C., Asplund, M., Grevesse, N., \& Sauval, A. J. 2006, A\&A, 456, 675 
Socas-Navarro, H. \& Norton, A. A. 2007, ApJL, 660, L153

Stein, R. F. \& Nordlund, A. 1998, ApJ, 499, 914

Suess, H. E. \& Urey, H. C. 1956, Reviews of Modern Physics, 28, 53

Turcotte, S., Richer, J., Michaud, G., Iglesias, C. A., \& Rogers, F. J. 1998, ApJ, 504, 539

Vernazza, J. E., Avrett, E. H., \& Loeser, R. 1976, ApJS, 30, 1

\section{Discussion}

S. Turck-ChIEzE: Among lot of other questions, I would like to know if the magnetic field and the variation are taken into account in the 3D solar atmosphere?

M. Asplund: In the 3D solar atmosphere model I described here that forms the basis of our solar abundance analysis, magnetic fields have not been considered, although the code can handle that. For spectral line formation in the quiet Sun magnetic fields are unimportant and thus this omission does not influence the conclusions regarding the chemical composition of the Sun.

J. Christensen-Dalsgandi: Two comments: (1) The slope of $\delta c / c$ is easy to understand: $\delta c / c \simeq 0$ in the convection zone ( $c^{2}$ is determined by the surface gravity), and in the radiation region is largest at the base of convection zone where the opacity change is largest. (2) The agreement between the new solar abundance and the abundance in the solar neighborhood is impressive, but the solar birth place is far from the present location and the composition could have been different there.

M. Asplund: (1) My point was mainly that even with the old, high solar abundances there is a discrepancy, although admittedly not nearly as large, immediately below the convection zone. This may be a coincidence of course, but it may also suggest that there are processes related to convection not yet taken into account in standard models (convection overshoot, internal gravity waves etc.)

(2) It is true of course that the Sun has migrated over the last 4.5 Gyr, but given its essentially perfectly circular orbit today it seems unlikely that it originated at a very different Galactocentric radius. Furthermore, all solar twins with the same stellar parameters as the Sun also have the same $\mathrm{O}$ abundance within the uncertainties. The same argument would then require that all of them also have migrated similarly.

HANS-G. LUDWIG: (1) What is the new abundance of nickel which you obtain? (2) What is your "best guess" value of $S_{H}$ ?

M. Asplund: (1) Our new 3D-based solar Ni abundance determination (Scott et al., in preparation) gives $\log \epsilon_{\mathrm{Ni}}=6.17 \pm 0.07$ using some ten Ni I lines.

(2) At this stage I don't have a preferred value for the scaling factor $S_{H}$ to the Drawin (1968) formula for inelastic H collisions. The available evidence from laboratory and quantum mechanical calculations for other elements imply that the Drawin formula overestimates the collisional cross-sections by several order of magnitudes, which suggests that $S_{H}=0$ also for O. The available center-to-limb observations for O I $777 \mathrm{~nm}$ on the other hand seem to suggest $S_{H} \simeq 1$. We clearly need quantum mechanical calculations for $\mathrm{O}$ I $+\mathrm{HI}$ collisions to resolve the issue but in the meantime we have obtained new and better UV observations for several O I and [O I] lines as well as spatially resolved spectra at different viewing angles $\mu$. 
S. KWOK: I just want to mention that interstellar medium lines have their own problems. In addition to dynamics and non-LTE effects, there are also uncertainties in excitation and ionization effects. In general, absorption lines are much easier to interpret than emission lines and their corresponding abundances are much more accurate.

M. AsPLUnD: I fully agree that ISM abundance analyses have their own problems and systematic errors, which only due to limited time I did not have the opportunity to discuss in any detail. 